\title{
Componentes epistemológicos para los trabajos de titulación en la Facultad de Ciencias Económicas y Negocios Universidad Tecnológica Equinoccial (UTE)
}

\author{
Epistemological components for degree Work, \\ at the School of Economics and Business - UTE
}

PATRICIO ANDINO SOSA

Universidad Tecnológica Equinoccial. Quito

patricio.andino@ute.edu.ec

RECIBIDO / Received: 4 de abril 2015

APROBADO / Accepted: 28 mayo del 2015

\section{Resumen}

Se presenta la experiencia en la implementación del Plan de mejora de los Trabajos de Titulación, realizado en la Facultad de Ciencias Económicas y Negocios de la Universidad Tecnológica Equinoccial - Quito. Ofrece una evolución histórica del proceso general de titulación y analiza los diversos problemas encontrados en la planificación y desarrollo de los trabajos de titulación. Incorpora las innovaciones implementadas, los comentarios y análisis formulados por estudiantes profesores y autoridades. La discusión aporta elementos de reflexión acerca del impacto de los formatos metodológicos de investigación en la calidad de los trabajos de titulación y estos a su vez, en los procesos de formación estudiantil, de acreditación institucional y de generación de conocimiento.

PALABRAS CLAVES: trabajos de titulación, Facultad de Ciencias Económicas y Negocios, UTE. 


\begin{abstract}
His paper aims to present an improvement in the implementation of the work Degree, held at the Business and Economics Sciences School of the Equinoctial Technological University, Quito - Ecuador. It presents a historical evolution of the overall certification process, and it analyzes the several problems that have arisen in the planning and development of the work degree. It has incorporated innovations that were implemented, the comments and analysis that were generated by teachers, students and academic directors. The discussion provides elements of reflection about the impact of methodological research formats, quality of work qualification, for the process of student training, institutional accreditation process and generation of knowledge.
\end{abstract}

KEYWORDS: work degree, Faculty of Economics and Business, UTE.

CLASIFICACIÓN JEL: A44 - Y40

\section{Introducción}

Uno de los factores de mayor influencia en la economía mundial contemporánea, es el acelerado desarrollo científico - tecnológico, cuyo resultado se traduce en innovadores productos y servicios con propuestas de valor que permiten a las organizaciones competir en mercados globales.

Para estimular el progreso innovador, es fundamental contar con una serie de factores claves, entre otros: grupos de investigadores experimentados, laboratorios con moderna infraestructura tecnológica, líneas de investigación definidas, financiamiento adecuado y claras políticas de investigación. A esto se suma la pertinencia universitaria, entendida, como la necesidad de enfocar las investigaciones en problemas surgidos de la realidad social del país.

Las Instituciones de Educación Superior (IES) juegan un papel clave en la generación de respues- tas a problemas sociales. Gran parte de la producción académica de un país es generada por docentes y estudiantes de las IES. Su decidida influencia en la producción de conocimiento, contribuye en la mejora de las condiciones económicas y culturales de la población.

Previa a su graduación de tercer nivel, los estudiantes universitarios ecuatorianos, pueden optar por un examen complexivo o por el desarrollo de un trabajo de titulación (tesina), al respecto, el Consejo de Educación Superior (CES) menciona en el Artículo 21 del Reglamento de Régimen Académico que:

Todo trabajo de titulación deberá consistir en una propuesta innovadora que contenga, como mínimo, una investigación exploratoria y diagnóstica, base conceptual, conclusiones y fuentes de consulta (...) el trabajo de titulación deberá guardar correspondencia con los aprendizajes 
adquiridos en la carrera y utilizar un nivel de argumentación coherente con las convenciones del campo del conocimiento (Consejo de Educación Superior, 2015, pág. 13).

Esta disposición procura que, dentro de cada IES, se generen procesos destinados a dar su cabal cumplimiento. Implica fortalecer la metodología de investigación científica y que, los componentes de la misma, sean manejados con propiedad por autoridades, docentes y estudiantes.

En función de los antecedentes anotados, se ha considerado reflexionar sobre la trascendencia del tema y recoger varias experiencias didácticas y administrativas que se han generado en la Facultad de Ciencias Económicas y Negocios (FCEN) de la Universidad Tecnológica Equinoccial - Matriz Quito.

La génesis de la FCEN data de 1971 con la creación del Instituto Tecnológico Equinoccial, en cuya oferta académica inicial constó la carrera "Dirigentes de Empresas". Para 1973 esta carrera se transformó en Ingeniería de Empresas. Como FCEN ha desarrollado sus actividades desde febrero de 1986, fecha en la cual fue reconocida la Universidad Tecnológica Equinoccial (UTE) por parte de las autoridades estatales de turno. (Vera, 2015).

Desde sus orígenes, las autoridades de la FCEN plantearon contar con una estructura metodológica que guíe los procesos de titulación. Una primera evaluación, acerca de este tema (Cevallos Gómez, 2012) identificó el comportamiento de los trabajos de titulación. Los más significativos resultados ${ }^{1}$ encontrados fueron:

1 Se analizaron 60 trabajos de titulación de la Carrera de Ingeniería en Empresas, sobre una población de 706 trabajos reportados desde principios de la década del 90, hasta el año 2012.

2 Revisión realizada en junio del 2014.
- $\quad$ El 40\% de los trabajos no definía correctamente el problema.

- $\quad$ El $23 \%$ no redactaba correctamente los objetivos generales y específicos

- $\quad$ El 33\% no formulaba hipótesis y, de los que la formularon, el $65 \%$ no la comprobaba empíricamente.

- El 44\% disponía de escasa información teórica cuando seleccionó su tema de investigación.

- $\quad$ El 47\% de los trabajos no observó normas de citación.

- Apenas un 7\% de las tesis arrojó emprendimientos.

Con estos resultados como base, la investigación se planteó conocer: ¿Cuál era para el año 2014 la situación de los trabajos de titulación en todas las carreras de la FCEN-UTE?

Al momento de realizar la investigación (2014) la FCEN contaba con las siguientes carreras: Ingeniería en Empresas y Negocios, Ingeniería en Comercio Exterior Integración y Aduanas, Ingeniería en Finanzas y Auditoría, Ingeniería en Marketing e Ingeniería en Talento Humano. A julio del 2014 la población era de 2019 estudiantes, 191 docentes (49 mujeres - 142 hombres), el $48 \%$ eran profesores a tiempo completo. (Vera, 2015).

La primera acción fue levantar un diagnóstico preliminar, tendiente a profundizar el problema a investigar. Cada uno de los miembros del colectivo docente de la materia Plan de Titulación, presentaron un reporte de experiencias acumuladas en sus clases y, en su condición de revisores y directores de trabajos de titulación. Se revisaron ${ }^{2}$ los reportes y se extrajeron las siguientes observaciones: 
- Inconsistencia metodológica en la presentación de trabajos, tanto de forma como de fondo. No se encontró una guía metodológica que oriente a maestros y estudiantes en la elaboración de las investigaciones; tampoco se encontró una modalidad de citación, ni de requisitos mínimos que debía cumplir el documento final. Cada docente proponía su propia estructura metodológica, según su propio criterio y experiencia. Por ende, había tantas opciones metodológicas como cuantos docentes laboraban en la FCEN.

- La existencia de una variedad de esquemas metodológicos generó que los diálogos entre estudiantes, directores y docentes evaluadores no siempre fuesen coincidentes. Situación por demás inoportuna que acarreó el reproceso permanente de los trabajos y, en la mayoría de casos, demoras excesivas e innecesarias. En algunos casos se requerían de parte de docentes evaluadores, que los estudiantes hagan replanteos o eliminación de capítulos enteros, lo que alteraba en forma parcial o total el sentido de la investigación.

- Las tesis arrojaron inconsistencias en su estructura epistemológica, lo cual evidenció, en general, una baja formación docente en metodología de la investigación científica. Otro elemento a considerar era que el claustro docente era muy heterogéneo en lo disciplinar, con orientación técnica profesional en diversas áreas del conocimiento, lo cual no garantizaba la existencia de un nivel homogéneo en fundamentos epistemológicos.

- La materia Plan de Titulación no ejercía una influencia significativa en los estudiantes, de cara a su proceso final de graduación; más bien, se la percibía como una asignatura de relleno. A esto habría que sumar el poco seguimiento que se le daba al proceso de graduación, lo cual ocasionó una tasa de graduación de apenas el 17\%.

El diagnóstico preliminar fue validado con un grupo de cincuenta docentes de la FCEN en tres reuniones de trabajo. Se analizaron los resultados del diagnóstico y se indagó acerca de los adicionales problemas que impedían a los docentes el desarrollo de los trabajos de titulación, identificándose los siguientes: escasas bases de datos para realizar las investigaciones, falta de tiempo para tutorías, desconocimiento de las líneas de investigación y escasa formación en algunos temas planteados. Se observó además: baja producción de publicaciones científicas, débil formación docente en metodología científica, poca participación en eventos científicos y escasas habilidades de gestores bibliográficos.

Del conjunto de hechos encontrados, se destacó la inexistencia de una metodología común para el proceso de investigación en trabajos de titulación en la FCEN. Se planteó como objetivo general el diseñar un proceso metodológico común para los trabajos de titulación en la FCEN y los mecanismos para su implementación.

Los objetivos específicos fueron:

- Identificar los principales problemas administrativos y metodológicos de los trabajos de titulación de la FCEN.

- Actualizar la propuesta metodológica para los trabajos de titulación de la FCEN.

3 Reunión realizada en septiembre de 2014 con la presencia de docentes de las carreras de la FCEN que, habían tenido experiencia en la Dirección de Trabajo de Titulación. 
- Implementar la metodología sugerida en todas las carreras de la FCEN.

Como novedad científica se presenta la implementación de un esquema epistemológico para las investigaciones de pregrado en una Facultad de Economía y Negocios.

\section{Metodología}

El nivel de la investigación fue exploratorio y descriptivo. Se usó modalidad de campo y docu- mental para la recolección de datos. La implementación de la propuesta en la FCEN, se abordó como proyecto de desarrollo.

Para identificar los principales problemas metodológicos de los trabajos de titulación, se determinó una población de estudio de 48 trabajos de titulación de todas las carreras de la FCEN de la ciudad de Quito, desde julio 2013 a julio 2014. Se tomó una muestra representativa de 22 trabajos $(45 \%)$, repartidos (Tabla 1) de la siguiente manera:

Tabla 1.

Composición muestra de investigación

\begin{tabular}{|c|c|c|c|c|c|c|}
\hline $\begin{array}{c}\text { Carreras FCEN- } \\
\text { UTE }\end{array}$ & Empresas & Finanzas & Marketing & $\begin{array}{c}\text { Comercio } \\
\text { Exterior }\end{array}$ & $\begin{array}{c}\text { Talento } \\
\text { Humano }\end{array}$ & Total \\
\hline \# Trabajos titulación & 4 & 4 & 4 & 7 & 3 & 22 \\
\hline
\end{tabular}

Elaboración: Autor

Para actualizar la propuesta metodológica de los trabajos de titulación, se revisaron formatos utilizados por 41 universidades nacionales y extranjeras. Se seleccionaron universidades nacionales con diferentes enfoques formativos, de tamaño diverso en número de alumnos y docentes.

En el caso de las universidades extranjeras se privilegió el análisis de universidades latinoamericanas. Se buscó escenarios sociales y culturales similares a los de la UTE. De igual manera se seleccionaron universidades con diferentes enfoques formativos, diverso tamaño en número de alumnos y carreras. (Ver Tabla 2). Los formatos de investigación fueron conseguidos de las páginas web de cada universidad, con la finalidad de identificar los componentes metodológicos y administrativos comunes.

La búsqueda documental se extendió a la revisión de libros de metodología de la investigación, de mayor uso en las universidades ecuatorianas (ver Tabla 3). 


\section{Tabla 2.}

Listado de universidades analizadas (formatos de titulación)

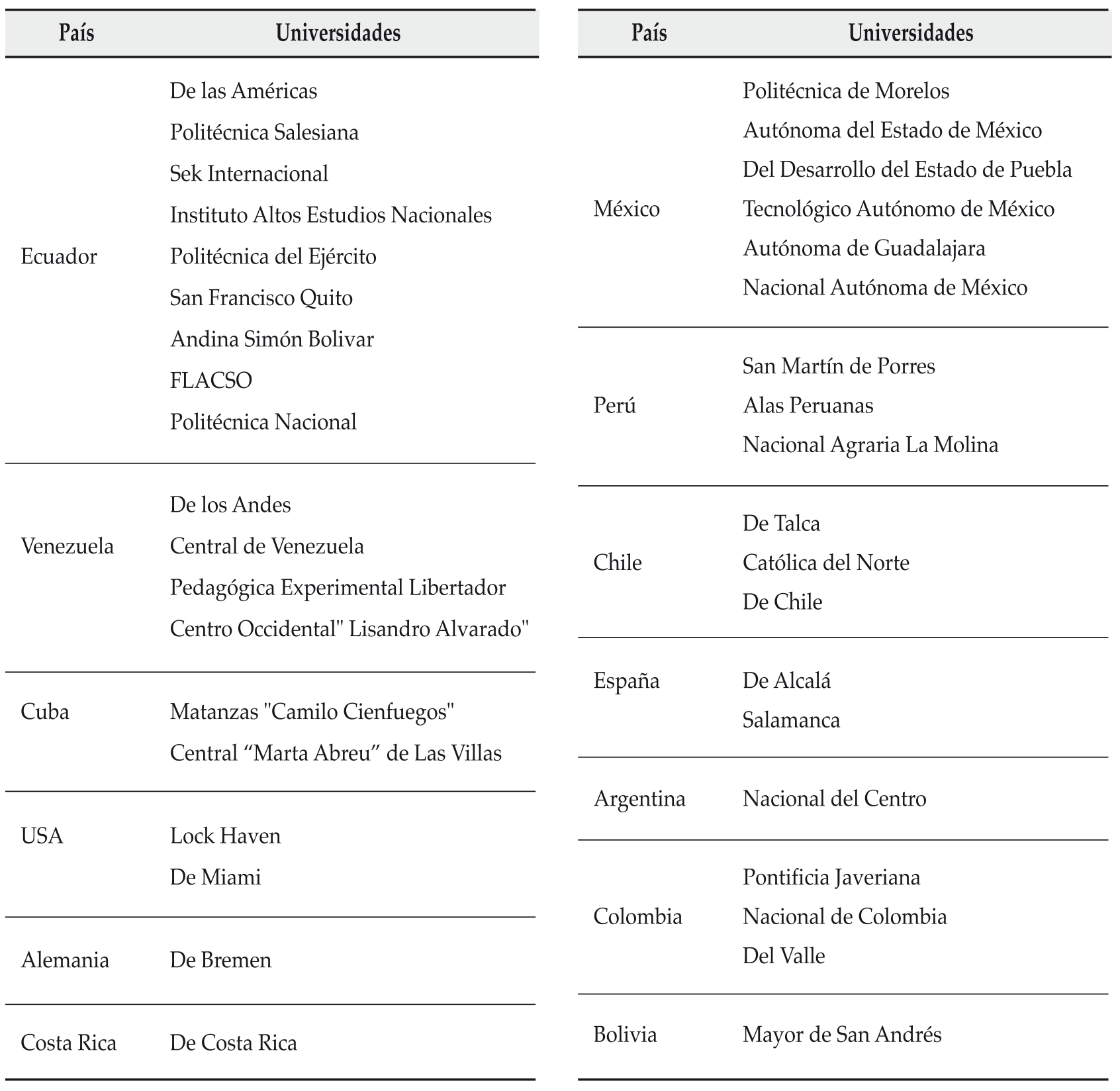


Tabla 3.

Listado de libros de metodología de la investigación analizados

\begin{tabular}{ll}
\hline \multicolumn{1}{c}{ Autor } & \multicolumn{1}{c}{ Título } \\
\hline $\begin{array}{l}\text { Academia Ciencias de } \\
\text { Cuba }\end{array}$ & $\begin{array}{l}\text { Metodología del Conocimiento } \\
\text { Científico }\end{array}$ \\
\hline Ander-Egg Ezequiel & Aprender a Investigar \\
\hline Andino Rubio Mauro & Investigación Social \\
\hline Arias Galicia Fernando & Metodología de la Investigación \\
\hline Bernal César & $\begin{array}{l}\text { Metodología de la Investigación } \\
\text { para Administración y Economía }\end{array}$ \\
\hline American Psychologi- & Manual de publicaciones APA \\
cal Association & \\
\hline Caballero Alejandro & Metodología Integral Innovadora \\
\hline Hernández Sampieri & Metodología de la Investigación \\
\hline Festinger y Katz & Métodos en Ciencias Sociales \\
\hline
\end{tabular}

\begin{tabular}{ll}
\hline \multicolumn{1}{c}{ Autor } & \multicolumn{1}{c}{ Título } \\
\hline $\begin{array}{l}\text { Ladrón de Guevara } \\
\text { Laureano }\end{array}$ & $\begin{array}{l}\text { Metodología de la Investi- } \\
\text { gación Científica }\end{array}$ \\
\hline Naupas Humberto, et.al & $\begin{array}{l}\text { Metodología de la Investi- } \\
\text { gación }\end{array}$ \\
\hline Rojas Soriano Raúl & $\begin{array}{l}\text { El proceso de la investigación } \\
\text { científica }\end{array}$ \\
\hline Tamayo Mario & $\begin{array}{l}\text { El proceso de la investigación } \\
\text { científica }\end{array}$ \\
\hline Tecla Alfredo & $\begin{array}{l}\text { Metodología en las Ciencias } \\
\text { Sociales }\end{array}$ \\
\hline Toro Iván y Parra Rubén & $\begin{array}{l}\text { Fundamentos epistemológicos } \\
\text { de la investigación y la } \\
\text { metodología de la investi- } \\
\text { gación }\end{array}$ \\
\hline Eco Umberto & Cómo se hace una tesis \\
\hline Bunge Mario & La Investigación Científica \\
\hline
\end{tabular}

Una segunda aplicación de la metodología de investigación se realizó a un grupo piloto de ocho estudiantes y luego se amplió a 255 estudiantes que habían terminado sus estudios, pero que, al mes de abril del 2015 no se titularon. Se aplicó un cronograma de trabajo que incluyó: convocatoria e inscripción de estudiantes; capacitación a estudiantes, maestros y personal administrativo.

\section{Resultados}

Se realizó una detallada revisión cualitativa de la estructura metodológica y de contenidos de los trabajos de titulación seleccionados. Luego se organizó la información con el fin de establecer los problemas más relevantes, encontrándose:

- Títulos excesivamente largos y mal estructurados;

- Falta de resumen y de palabras claves al inicio del documento;

- Insuficiente construcción del Marco Teórico ;

- Ausencia del problema de investigación;

- Inadecuada selección de los verbos para la conformación de los objetivos; 
- Estructura muy extensa del objetivo general, perdiéndose a veces el propósito a cumplir;

- Objetivos generales divorciados de la naturaleza de la investigación, con aportes que no guían sustancialmente su ejecución;

- Exceso de objetivos específicos, en su mayoría, sin relación alguna con el objetivo general ;

- Hipótesis mal formuladas, inadecuadamente justificadas y en número excesivo;

- Variables mal definidas y en número abundante;

- Muy débil o inexistente operacionalización de variables;

- Débil o inexistente articulación entre componentes claves de investigación: título, problema, objetivo general. Hipótesis, variables y marco teórico;

- $\quad$ Excesivo número de páginas (algunas sobrepasan las 200);

- Desproporción entre los temas del trabajo de graduación planteados y el nivel académico de los estudiantes de pregrado.

Los problemas encontrados fueron analizados en primera instancia por el colectivo docente ${ }^{4}$ de Plan de Titulación de la FCEN. El grupo formuló varias opciones de reforma, las cuales fueron planteadas al Decanato de la FCEN. Entre las principales recomendaciones se mencionaron:

- Los temas de investigación deben guardar estrecha correspondencia con las líneas de investigación de cada carrera y con el Plan Nacional del Buen Vivir.

\footnotetext{
4 Docentes: Patricio Andino Sosa, Francisco Jara y Rogelio Suárez.
}

- Estructurar un formato metodológico general, el cual recoja los principales lineamientos a seguir por docentes y estudiantes.

- Replantear el sílabo y los contenidos de la asignatura Plan de Titulación, para que recoja los lineamientos del nuevo formato metodológico a desarrollar.

- Capacitar a los docentes acerca del nuevo formato metodológico, sobre escritura académica, científica y normas APA.

- Indexar la Revista Economía y Negocios en Latindex, como elemento motivador para publicar investigaciones relevantes de docentes y estudiantes.

- Examinar la incorporación o exclusión de determinados componentes teóricos en el formato metodológico de investigación, para que se ajusten al nivel de investigaciones que realizan los estudiantes de pregrado de la FCEN.

- Conformar el Centro de Investigaciones de la FCEN, el cual estará constituido por grupos de investigadores, para que ellos, entre otras actividades, sean los que desarrollen las líneas de investigación y propongan temas.

- Incorporar desde el principio del trabajo de titulación al director y evaluadores, con la finalidad que los estudiantes tengan la asesoría oportuna en todo el proceso.

- Implementar "Predefensa del trabajo" cuyo propósito sería hacer una presentación pública de los resultados obtenidos y discutirlos antes de la defensa oral.

- Plantear varias reformas en el Reglamento de Trabajos de Titulación de la UTE, que recoja los lineamientos recomendados. 
Figura 1.

Proceso general de investigación para los trabajos de titulación

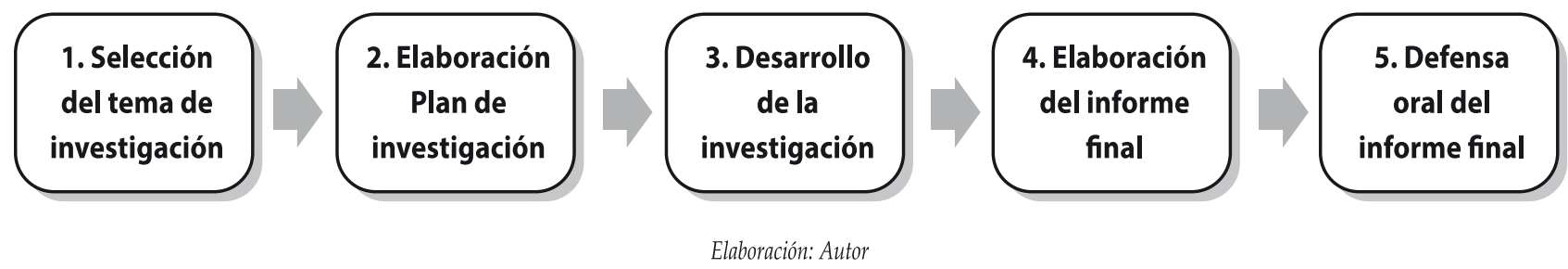

La revisión documental de los formatos de trabajos de investigación de las universidades seleccionadas y de los libros de texto de epistemología, arrojó un proceso general a seguir. (Figura 1)

Una búsqueda a mayor profundidad, aportó resultados acerca de los componentes que debían ser parte constitutiva del informe final. Los documentos de universidades y textos revisados, presentaron múltiples opciones de trabajo, en función del tipo de carre$\mathrm{ra}$, concepciones y procedimientos recomendados y, sin embargo, se encontraron grandes bloques temáti$\cos$, los cuales se fueron organizando en orden precedente.

Los bloques encontrados se armaron con la finalidad de cumplir con la metodología general de investigación, ya que "los componentes del diseño teórico en su lógica interna estructural y conceptual son únicos para todas las ciencias" (Guardo \& Fajardo, 2009, pág. 4).

Para dotar al informe final del trabajo de titulación, de un formato internacional estándar, se establecieron cuatro capítulos en función del formato IMRyD (Introducción, Método, Resultados y Discusión) utilizado por la generalidad de revistas científicas. A este formato se acoplaron las recomendaciones de las Normas APA (American Psychological Association, 2010) las cuales son de amplia utilización dentro del ámbito de las Ciencias Sociales.
La FCEN generó un Manual para la presentación de Trabajos de Titulación (Andino \& Jara, 2014), el cual recogió los principales lineamientos de las Normas APA (6ta. Edición ,3ra. En español). Al estar la normativa APA estructurada para el ámbito de los Estados Unidos de América, se consideró necesario adecuarla a nuestro medio.

El formato del informe final propuesto (Figura 2) se compone por una fase teórica $(40 \%)$ y otra empírica o práctica $(60 \%)$; situación que tiene como propósito evitar informes exclusivamente teóricos, dando peso significativo a los resultados que aporten a la solución del problema planteado.

Se estudiaron los porcentajes (en número de páginas) aproximados que cada una de las propuestas de las diversas universidades y textos de enseñanza recomendaban. En la mayoría eran valores referenciales, pero en otros casos se señalaba con exactitud el número de páginas o palabras a usar. Esto resultó de vital importancia ya que en la investigación inicial, los alumnos de la FCEN no tenían una ponderación establecida, encontrándose trabajos en los cuales el marco teórico abarcaba el 70\% del trabajo, o resultados que conformaban el $80 \%$ del informe. La estructura general y los pesos se definieron según consta en la Figura 2.

La extensión que debía tomar el informe final fue otro aspecto que se valoró, encontrando que los 
Figura 2.

Componentes ponderados del Trabajo de Titulación

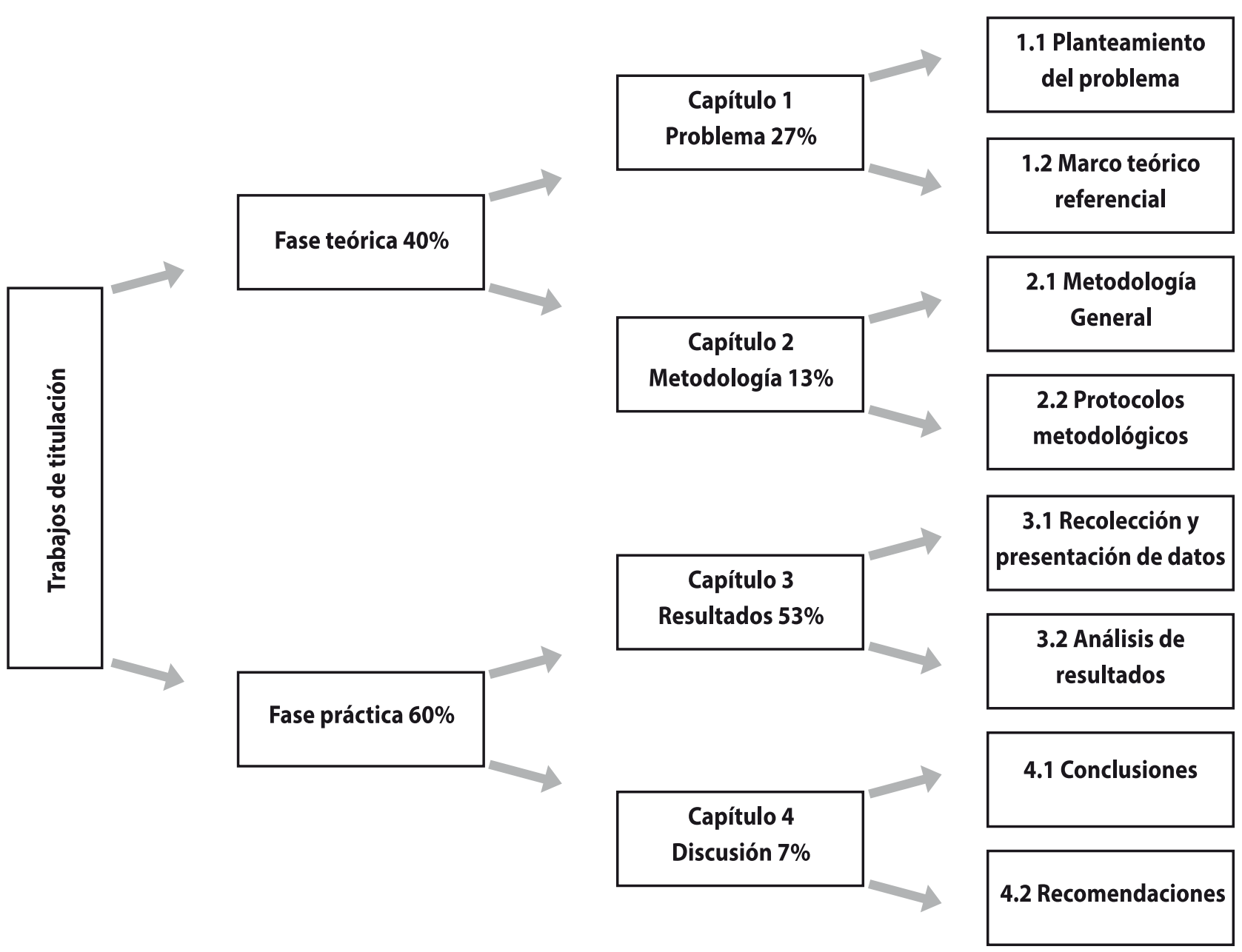

formatos evaluados recomendaban la utilización de entre 60 a 120 páginas. Para la FCEN, se propuso un mínimo de 60 y un máximo de 80 páginas. El criterio dominante fue priorizar la calidad antes que la cantidad de páginas del informe. En lo referente a los Anexos, se recomendó máximo el 25\% del número de páginas presentadas en el cuerpo del documento. Cabe señalar que los porcentajes señalados tuvieron la intencionalidad de proporcionar un equilibrio en la elaboración del informe final.
Otro criterio valorado fue el número de referencias bibliográficas a utilizar. No se encontró en la documentación analizada, una tendencia en este tema. Hay universidades que no reseñan recomendación alguna, hay otras que, proponen entre 12 a 40 referencias. Para la FCEN se determinó que en el informe final consten mínimo 20 referencias bibliográficas, de las cuales, el 50\% deben ser artículos científicos. Para completar este objetivo, se armó la estructura de cada uno de los componentes. Se siguió el criterio de 
Tabla 4.

Estructura del Capítulo 1- Informe final trabajo de titulación

\begin{tabular}{|c|c|c|c|c|c|c|}
\hline \multicolumn{6}{|c|}{ CAPÍTULO 1. INTRODUCCIÓN - ¿Cuál es el problema? ¿Por qué es importante? } & $\begin{array}{l}\text { \# páginas } \\
\text { recomen- } \\
\text { dadas }\end{array}$ \\
\hline \multirow{14}{*}{ 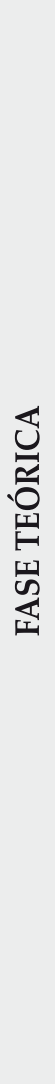 } & \multirow{11}{*}{\multicolumn{2}{|c|}{ coblema }} & 1.1.1 & Problema a investigar & Señalar el problema a investigar & \multirow{11}{*}{7 a 10} \\
\hline & & & 1.1 .2 & Objeto de estudio teórico & Identificar objeto teórico a estudiar & \\
\hline & & & 1.1 .3 & Objeto de estudio práctico & Identificar objeto práctico a estudiar & \\
\hline & & & \multirow{3}{*}{1.1 .4} & \multirow{3}{*}{ Planteamiento del problema } & Diagnóstico: síntomas y causas del problema & \\
\hline & & & & & Pronóstico: escenario negativo del problema & \\
\hline & & & & & $\begin{array}{l}\text { Control del Pronóstico: escenario positivo del } \\
\text { problema }\end{array}$ & \\
\hline & & & 1.1 .5 & Formulación del problema & Formular preguntas de investigación & \\
\hline & & & 1.1 .6 & Sistematización del problema & $\begin{array}{l}\text { Presentar de } 3 \text { a } 5 \text { subpreguntas de investi- } \\
\text { gación }\end{array}$ & \\
\hline & & & 1.1.7 & Objetivo general & Es el propósito de estudio. Señalar un objetivo & \\
\hline & & & $1-1.8$ & Objetivos específicos & $\begin{array}{l}\text { Surgen del objetivo general. Señalar de } 3 \text { a } 5 \\
\text { actividades a ejecutar }\end{array}$ & \\
\hline & & & 1.1 .9 & Justificaciones & $\begin{array}{l}\text { Detallar las razones para hacer la investi- } \\
\text { gación }\end{array}$ & \\
\hline & & Marco & 1.2 .1 & Marco teórico & $\begin{array}{l}\text { Fundamentación teórica de la investigación } \\
\text { Revisión bibliográfica }\end{array}$ & 8 a 10 \\
\hline & & referencial & 1.2 .2 & Marco conceptual (opcional) & Máximo 4 conceptos clave & \\
\hline & & & & Total Capítu & 101 & 15 a 20 \\
\hline
\end{tabular}

Elaboración: Autor

agrupar por temáticas y ver las coincidencias que presentaban cada una de las propuestas recogidas en los diversos documentos revisados.

En el Capítulo 1 (Ver Tabla 4) se pone particular interés en establecer claramente el problema de investigación y los objetos de estudio a trabajar. Se da un peso significativo al planteamiento del problema, para ubicar los antecedentes que contextualizan la investigación. El marco teórico se estructura en función de los objetos de estudio propuestos, tratando en lo posible de darle un peso equitativo a cada uno de ellos. Se incorporó como elemento guía para los estudiantes, el número de páginas referenciales que debe abarcar cada uno de los epígrafes señalados. Recalcando que son valores netamente referenciales.

El Capítulo 2 (Tabla 5), es el que más modificaciones incorporó. Inicia con el estudio de los aspectos metodológicos clásicos, los de mayor familiaridad para profesores y estudiantes y presentes en la generalidad de los trabajos de titulación. A esta sección se le ha denominado "Metodología General". En segunda instancia se propuso el concepto "Protocolos metodológicos" con la finalidad que los estudiantes describan las actividades lógicas y secuenciales que deben realizar para dar cumplimiento a los objetivos específicos. 
Tabla 5.

Estructura del Capítulo 2 - Metodología

\begin{tabular}{|c|c|c|c|c|c|c|}
\hline \multicolumn{6}{|c|}{ CAPÍTULO 2. METODOLOGÍA - ¿Cómo investigo el problema? } & $\begin{array}{l}\text { \# páginas } \\
\text { recomen- }\end{array}$ \\
\hline \multirow{6}{*}{\multicolumn{2}{|c|}{ 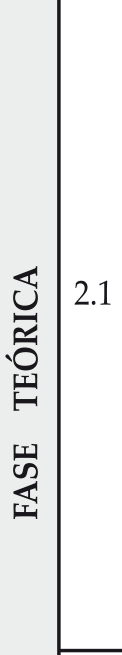 }} & \multirow{6}{*}{$\begin{array}{l}\text { Metodología } \\
\text { general }\end{array}$} & 2.1 .1 & Nivel de estudio & $\begin{array}{l}\text { Fundamentar si la investigación es explorato- } \\
\text { ria, descriptiva o de cualquier otro nivel. }\end{array}$ & \multirow{7}{*}{3 a 5} \\
\hline & & & 2.1 .2 & Modalidad de investigación & $\begin{array}{l}\text { Identificar si es de campo, documental o } \\
\text { proyecto de desarrollo. }\end{array}$ & \\
\hline & & & 2.1 .3 & Método & $\begin{array}{l}\text { Seleccionar si es inductivo-deductivo; analítico } \\
\text { sintético o cualquier otro método }\end{array}$ & \\
\hline & & & 2.1 .4 & $\begin{array}{l}\text { Población y muestra (en } \\
\text { caso de aplicar) }\end{array}$ & $\begin{array}{l}\text { Definir método de muestreo y número de } \\
\text { participantes }\end{array}$ & \\
\hline & & & 2.1 .5 & $\begin{array}{l}\text { Selección instrumentos de } \\
\text { investigación }\end{array}$ & $\begin{array}{l}\text { Elegir entre: observación, encuestas, entrevis- } \\
\text { tas, análisis de documentos o cualquier otro } \\
\text { instrumento }\end{array}$ & \\
\hline & & & 2.1.6 & Procesamiento de datos & $\begin{array}{l}\text { Señalar la forma de clasificar, registrar y } \\
\text { tabular los datos }\end{array}$ & \\
\hline & 2.2 & $\begin{array}{l}\text { Protocolos } \\
\text { metodológi- } \\
\text { cos }\end{array}$ & \multicolumn{3}{|c|}{$\begin{array}{l}\text { Detalla las actividades lógicas y secuenciales que deben ejecutarse para cumplir con } \\
\text { cada uno de los objetivos específicos. Su ejecución arroja resultados que contribuyen } \\
\text { a cumplir con el objetivo general. }\end{array}$} & \\
\hline
\end{tabular}

Elaboración: Autor

Tabla 6.

Composición de los Capítulos 3 y 4

\begin{tabular}{|c|c|c|c|c|}
\hline \multicolumn{4}{|c|}{ CAPÍTULO 3. RESULTADOS - ¿Cuáles fueron los hallazgos? } & $\begin{array}{l}\text { \# páginas } \\
\text { recomen- } \\
\text { dadas }\end{array}$ \\
\hline \multirow{5}{*}{ 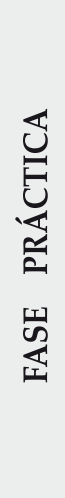 } & 3.1 & $\begin{array}{l}\text { Recolección y tratamiento } \\
\text { de datos }\end{array}$ & $\begin{array}{l}\text { Implementación de los protocolos metodológicos. } \\
\text { Obtención de resultados específicos }\end{array}$ & \multirow{2}{*}{30 a 40} \\
\hline & 3.2 & $\begin{array}{l}\text { Presentación y análisis de } \\
\text { resultados }\end{array}$ & $\begin{array}{l}\text { Detalle de los resultados obtenidos de los protocolos } \\
\text { metodológicos }\end{array}$ & \\
\hline & \multicolumn{3}{|c|}{ CAPÍTULO 4. DISCUSIÓN - ¿Qué significan los resultados? } & $\begin{array}{l}\text { \# páginas } \\
\text { recomen- } \\
\text { dadas }\end{array}$ \\
\hline & 4.1 & Conclusiones & $\begin{array}{l}\text { Explicar como los resultados contribuyen a responder la } \\
\text { pregunta de investigación }\end{array}$ & \multirow{2}{*}{3 a 5} \\
\hline & 4.2 & Recomendaciones & $\begin{array}{l}\text { Detalla los planes de acción o aplicaciones futuras acerca } \\
\text { de la investigación }\end{array}$ & \\
\hline
\end{tabular}

Elaboración: Autor 
Los Capítulos 3 y 4 se plantean como una consecuencia lógica de los capítulos anteriores. El propósito fundamental es que en el "tratamiento y presentación de resultados" se detallen todos los resultados obtenidos, como consecuencia de aplicar una metodología general y los protocolos metodológicos respectivos.

Cabe señalar que se han desarrollado materiales de apoyo para todos los puntos contenidos en el formato respectivo. Estos materiales han sido socializados con estudiantes, docentes y autoridades.

Las recomendaciones aquí planteadas, fueron implementadas gradualmente en la FCEN desde marzo del 2014 a julio del 2015. Se inició con una prueba piloto de 72 estudiantes de la Carrera de Comercio Exterior, Integración y Aduanas que tomaron la materia de Plan de Titulación en el semestre de marzo a julio 2014. Terminado el período, junto con el Colectivo de Docentes de la materia Plan de Titulación se evaluaron las acciones iniciales y se procedió a realizar varios ajustes. Para el período septiembre 2014 a enero 2015, se trabajó con 153 estudiantes de todas las carreras de la FCEN, dentro de la materia Plan de Titulación, para finalmente, en el período marzo-julio 2015 se trabajaron con 180 alumnos. El detalle consta en la Tabla 7.

Tabla 7.

Implementación de nueva propuesta de trabajos de titulación

\begin{tabular}{|l|l|c|c|c|}
\hline \multicolumn{1}{|c|}{ Modalidad } & \multicolumn{1}{c|}{ Período académco } & \# Estudiantes & \# Profesores & \# Carreras \\
\hline Prueba piloto & Marzo a julio 2014 & 45 & 1 & 1 \\
\hline Implementación 1 & Sept. 2014 a enero 2015 & 122 & 2 & 2 \\
\hline Implementación 2 & Marzo a julio 2015 & 168 & 4 & 5 \\
\hline & Total & 335 & 4 & 5 \\
\hline
\end{tabular}

Elaboración: Autor

Con la finalidad de validar el procedimiento para los trabajos de titulación, se laboró con un grupo piloto de ocho estudiantes de la carrera de Comercio Exterior, Integración y Aduanas. El total de estudiantes participantes tenían aprobado su plan de investigación, pero la mitad no tenían tutor. 
Tabla 8.

Composición de actividades para trabajos de titulación

\begin{tabular}{lll}
\hline Actividad & \multicolumn{1}{c}{ Involucrados } & Plazo (en semanas) \\
\hline Revisión de Plan & Estudiante/Director & 1 \\
\hline Redacción final capítulos I y II & Estudiante/Director & 4 \\
\hline Revisión capítulos I y II & Calificadores & 1 \\
\hline Redacción final capítulo III & Estudiante/Director & 6 \\
\hline Redacción final capítulo IV & Estudiante/Director & 2 \\
\hline Revisión capítulos III y IV & Calificadores & 2 \\
\hline Redacción Informe final & Estudiante/Director & 1 \\
\hline Calificación informe final & Calificadores & 2 \\
\hline Presentación aptitud legal & Estudiante & 2 \\
\hline
\end{tabular}

Elaborado por: Patricio Andino Sosa (2015)

Se estructuraron una serie de actividades tendientes a darle una fluidez metodológica al desarrollo de las investigaciones (Tabla 8). La novedad radicó en armar un proceso de capacitación que incluyó a docentes, docentes tutores y docentes calificadores. Por ejemplo, para el desarrollo del Capítulo 1 se dio una charla de tres horas a los docentes tutores para establecer los componentes básicos del capítulo, cuya finalidad era tener puntos comunes de trabajo.

La capacitación con los estudiantes siguió los mismos propósitos que la de los docentes, la única diferencia era que se utilizaron cuatro horas de trabajo. Tanto la capacitación con docentes y estudiantes, tuvo como base la metodología descrita en este documento.

Los resultados de la prueba piloto muestran (Tabla 9) que todos los estudiantes se graduaron. La mitad de ellos se graduaron en los tiempos planificados y la otra mitad hasta 9 semanas después de lo planificado. La nota promedio de graduación fue de $9,2 / 10$

Tabla 9.

Tiempos de graduación de estudiantes

\begin{tabular}{rc}
\hline Tiempo de graduación & $\begin{array}{c}\text { Estudiantes } \\
\text { graduados }\end{array}$ \\
\hline Graduado en 21 semanas & 4 \\
\hline Graduado en 23 semanas & 2 \\
\hline Graduado en 25 semanas & 1 \\
\hline Graduado en 30 semanas & 1 \\
\hline TOTAL & $\mathbf{8}$ \\
\hline
\end{tabular}

Elaborado por: Patricio Andino 
Finalmente, los resultados de la prueba piloto se llevaron a un grupo de 272 estudiantes que habían terminado sus estudios, pero no se titularon. La Tabla 10 detalla la composición del grupo que optó por hacer trabajos de titulación.

Tabla 10.

Participación de estudiantes

\begin{tabular}{rcc}
\hline Situación & \# Estudiantes & Porcentaje \\
\hline Trabajo de titulación & 255 & $93,8 \%$ \\
\hline Examen complexivo & 17 & $6,3 \%$ \\
\hline TOTAL & $\mathbf{2 7 2}$ & $\mathbf{1 0 0 \%}$ \\
\hline
\end{tabular}

Elaboración: Patricio Andino
Del grupo total, 255 estudiantes escogieron realizar trabajos de titulación. Para esto, se invitó a los estudiantes a inscribirse. La Tabla 11 muestra el estado en el cual se encontraban al inicio del proceso, se puede notar que el 75,7 no tenía tutor ni título de investigación. Se realizó un seguimiento similar que al grupo piloto, pese a las propias complejidades de manejar un grupo de mayor cantidad de estudiantes ${ }^{5}$.

Vale destacar el crecimiento mostrado en todos los porcentajes de las situaciones que se trataron, sobre todo, el avance del 50\% de los estudiantes que empezaron sus trabajos de titulación.

Tabla 11.

Estados de los trabajos de titulación

\begin{tabular}{lccccc}
\hline $\begin{array}{c}\text { Situación del trabajo } \\
\text { de titulación }\end{array}$ & \multicolumn{2}{c}{ Situación inicial } & & \multicolumn{2}{c}{ Situación inicial } \\
\cline { 3 - 5 } Terminado. Pendiente la defensa oral & 2 & $0,8 \%$ & & Porcentaje \\
\hline En proceso de revisión de calificadores & 5 & Porcentaje & & $27,0 \%$ & $10,6 \%$ \\
\hline Avance menor al 50\% & 36 & $14,1 \%$ & 26 & $10,2 \%$ \\
\hline Avance del 51\% al 95\% & 19 & $7,5 \%$ & 128 & $50,2 \%$ \\
\hline Sin tutor ni título & 193 & $75,7 \%$ & 51 & $20,0 \%$ \\
\hline & TOTAL & $\mathbf{2 5 5}$ & $\mathbf{1 0 0 , 0 \%}$ & 23 & $9,0 \%$ \\
\hline
\end{tabular}

Elaboración: Patricio Andino

5 La coordinación del trabajo estuvo a cargo de la Ing. Patricia Vargas, junto con un grupo de cinco pasantes de la Carrera de Comercio Exterior, Integración y Aduanas. 


\section{Discusión}

Luego de la implementación del esquema de trabajo metodológico en la FCEN, podemos mencionar lo siguiente:

El impacto en los estudiantes, al contar con un esquema claro de trabajo, fue muy positivo; lo que ha permitido que se dinamice de manera importante el número de estudiantes que se encuentran inmersos en su trabajo de titulación. Mencionaron los estudiantes que se facilitó el desarrollo de su investigación, al contar con un esquema metodológico unificado para que directores y evaluadores tengan la misma guía.

Los estudiantes que trabajaron con el nuevo esquema metodológico, bajaron de manera significativa el tiempo de la investigación, lo que generó que los trabajos desarrollados se entreguen en los tiempos establecidos y con mayor calidad académica.

Si bien es cierto la mayoría de docentes mostró interés en trabajar con el nuevo esquema de trabajo, su adopción fue lenta. Se observó la generación de un diálogo enriquecedor entre docentes, y sobre todo, entre docentes y estudiantes. Hay que mencionar que pocos docentes, por una diversidad de causas, se negaron a usar el esquema nuevo.

Una vez se fueron evidenciando los resultados, se trabajó paralelamente en sugerencias a ser consideradas en el Reglamento de Titulación de la UTE. La magnitud del trabajo, sin embargo,

6 Resolución No 05-CU-UTE-2015 del 28 de enero del 2015.

7 Resolución Rectoral No.-43-R-UTE-2015 del 1 de junio del 2015, mediante la cual el Ing. José Julio Cevallos, Rector de la UTE, aprueba el funcionamiento del Centro de Investigaciones Administrativas y Económicas (CIADE) de la Facultad de Ciencias Económicas y Negocios. hizo imprescindible hacer una Reforma integral del mencionado Reglamento, el cual fue aprobado por Consejo Universitario ${ }^{6}$.

El familiarizarse con una estructura metodológica contribuyó a que los docentes generen investigaciones propias que devinieron en artículos científicos. Para dar cabida a varias propuestas se indexó a la Revista Economía y Negocios en Latindex. Algunas reflexiones de docentes de la FCEN ya han sido incorporadas a la revista.

Al implementar el nuevo diseño metodológico, se lograron otros resultados importantes que no estaban inicialmente planteados. Al reformular una serie de actividades investigativas de la FCEN, se tuvo que repensar las líneas de investigación vigentes. Al tratar de organizar los procesos de investigación, se vio la necesidad de reactivar el Centro de Investigaciones Económicas ${ }^{7}$ de la FCEN.

El contar con un esquema metodológico de trabajo, plenamente validado y en ejecución, ha constituido un valioso impulso dinamizador de las actividades relacionadas con los procesos de titulación. Los estudiantes han sido los más receptivos a este cambio, quedando pendiente aún la integración mayoritaria de docentes.

El mejorar los procesos de titulación tiene un efecto muy positivo, de cara a elevar de manera significativa el porcentaje de graduados en todas las carreras de la FCEN, permitiendo con esto, presentar indicadores sólidos para Acreditación.

Los estudiantes de la FCEN, al usar la nueva metodología de trabajos de titulación, tienen que desarrollar una visión que excede lo exclusivamente coyuntural de su carrera y les invita a desarrollar una visión más integral de las organizaciones y su entorno. Al trabajar en ambientes multi e interdisciplinarios, se fortalecen las concepciones holísticas, lo cual enriquece los contenidos de los trabajos de titulación. 
Al constituir el Trabajo de Titulación un importante subproceso dentro del proceso general de formación, y, al ser la epistemología, la base central del conocimiento científico, se puede afirmar que se ha contribuido positivamente a una mejor comprensión y aplicación de la epistemología, bajándola de una concepción de "halo misterioso" de "piedra filosofal" en que la han puesto algunos docentes y estudiantes $\mathrm{y}$, más bien, ubicándola en una posición más terrenal, más práctica a la hora de asegurar sólidos resultados de aprendizaje.

Se incorporó al docente director y a los evaluadores desde el principio del proceso, para que asesoren desde un inicio a los estudiantes y, de esta manera, evitar reprocesos lamentables.

Se impulsó el rol activo de los estudiantes en la identificación de problemas originados en necesidades de la práctica social, para de esta manera, fortalecer el conocimiento de los síntomas y causas de la realidad ecuatoriana; con la intencionalidad que el estudiante vaya familiarizándose en la generación de soluciones, ante problemas presentados en su entorno.

Los principales lineamientos de trabajo y las reflexiones generadas, influyeron vigorosamente en la concreción de un nuevo Reglamento de Titulación para la UTE, la conformación del Centro de Investigaciones de la Facultad, acompañadas de una nueva estructura de las líneas de investigación.

Los resultados obtenidos corresponden a la implementación en una Facultad de Ciencias Económicas y Negocios, y si bien la metodología de la ciencia es general, habrá que tomar las debidas precauciones a la hora de aplicarlo en otros contextos.

\section{Referencias Bibliográficas}

- Academia de Ciencias de Cuba. (1975). Metodología del conocimiento científico. La Habana: Editorial Ciencias Sociales.

- American Psychological Association. (2010). Manual de Publicaciones APA. México: Moderno.

- Ander- Egg, E. (2012). Aprender a investigar. Quito: Miraflores.

- Andino Rubio, P. (1994). Investigación Social. Latacunga: Alvarado.

- Andino, P., \& Jara, F. (2014). Normas para la presentación de trabajos de titulación. Quito: Facultad de Ciencias Económicas y Negocios UTE.

- Arias Galicia, F. (2012). Metodología de la Investigación. México: Trillas.

- Bernal, C. (2006). Metodología de la Investigación. México: pearson.

- Bunge, M. (1976). La Investigación Científica. Barcelona: Seix y Barral. 
- Caballero Romero, A. (2013). Metodología Integral Innovadora para planes y tesis. México: Cengage.

- Cevallos Gómez, J. (2012). Universidad e Investigación. Una reflexión sobre los trabajos de investigación. Quito: Mentall.

- Consejo de Educación Superior. (10 de junio de 2015). Reglamento de Régimen Acádemico. Quito: CES.

- Eco, U. (1977). Cómo se hace una tesis. Guanajato: Gedisa.

- Escuela Politécnica Nacional del Ecuador. (2015). www.epn.edu.ec. Obtenido de http:/ / www.epn.edu.ec/ investigacion/repositorio-documental/\# reglamentos

- Facultad Latinoamericana de Ciencias Sociales- Sede Ecuador. (2015). www.flacso.edu.ec. Obtenido de https:/ / www.flacso.edu.ec/portal/docencia/investigador/

- Festinger, L., \& Katz, D. (1992). Los métodos de la investigación social. Buenos Aires: Paidos.

- Guardo, M., \& Fajardo, M. (2009). Los componentes del diseño teórico de la investigación científica. Una reflexión praxiológica. Pedagogía Universitaria, XIV(3), 1-28.

- Hernández Sampieri, R., Fernández Collado, C., \& Baptista Lucio, P. (2014). Metodología de la Investigación. México: McGraw Hill.

- Instituto de Altos Estudios Nacionales. (2015). www.iaen.edu.ec. Obtenido de http:/ / www.iaen.edu.ec/ universidad/docentes-investigadores /

- Instituto Tecnológico Autónomo de México. (2014).https://www.itam.mx/. Obtenido de http:// caie.itam.mx/

- Ladrón de Guevara, L. (1978). Metodología de la investigación científica. Bogotá: Universidad Santo Tomás.

- Lock Haven University. (2015). http://www.lhup.edu/. Obtenido de http://www.lhup.edu/About/policies/

- Ñaupas, H., Mejía, E., Novoa, E., \& Villagómez, A. (2014). Metodología de la investigación. Bogotá: Ediciones de la U.

- Pontificia Universidad Javeriana. (2015). http://www.javeriana.edu.co/. Obtenido de http:/ / www. javeriana.edu.co/investigacion

- Tamayo , M. (1986). El proceso de la investigación científica. México: Limusa.

- Tecla, A. (1978). Metodología en las Ciencias Sociales. México: Ediciones Taller Abierto.

- Toro Jaramillo, I., \& Parra Ramirez, D. (2010). Fundamentos epistemológicos de la investigación y la metodología de la investigación. Medellin: Editorial EAFIT.

- Universidad Agraria La Molina. (2014). http://www.lamolina.edu.pe/. Obtenido de http://www. lamolina.edu.pe/investigacion/

- Universidad Alas Peruanas. (2014). http://www.uap.edu.pe/. Obtenido de http://www.uap.edu.pe/Esp/ CentrosdeProduccion/Inicio.aspx 
- Universidad Andina Simón Bolivar- Sede Ecuador. (2015). www.uasb.edu.ec. Obtenido de http://www.uasb. edu.ec/web/guest/investigacion

- Universidad Autónoma del Estado de México. (2015). http://www.uaemex.mx/. Obtenido de http:/ / www. uaemex.mx/index.php/bienvenida

- Universidad Católica del Norte - Chile. (2015). http://www.ucn.cl/. Obtenido de http:/ / www.ucn.cl/ investigacion/

- Universidad Central de Venezuela. (2014). www.ucv.ve. Obtenido de http:// www.ucv.ve/investigacion/ institutos.html

- Universidad Central Martha Abreu- Cuba. (2014). http://www.uclv.edu.cu/. Obtenido de http:// www.uclv. edu.cu/investigacion/ centros-de-investigacion/

- Universidad Centroccidental Lisandro Alvarado - Venezuela. (2014). http://www.ucla.edu.ve/. Obtenido de http:/ / cdcht.ucla.edu.ve/investigacion/

- Universidad de Chile. (2014). http://www.uchile.cl/. Obtenido de http:// www.uchile.cl/investigacion

- Universidad de Costa Rica. (2015). https://www.ucr.ac.cr/. Obtenido de http:/ / www.vinv.ucr.ac.cr/

- Universidad de las Américas - Quito. (2015). www.udla.edu.ec. Obtenido de http:/ / www.udla.edu.ec/ investigaciones/proyectos/

- Universidad de las Fuerzas Armadas ESPE. (2015). www.espe.edu.ec. Obtenido de http:/ / www.espe.edu.ec/ ?q=investigacion/pol\%C3\%ADticas-y-1\%C3\%ADneas-de-investigaci\%C3\%B3n

- Universidad de los Andes - Venezuela. (2015). www.ula.ve. Obtenido de http:/ / www2.ula.ve/cdcht/index. php?option=com_content\&task=view\&id=256\&Itemid $=237$

- Universidad de Matanzas. Camilo Cienfuegos. (2015). http://www.umcc.cu/. Obtenido de http:// www.umcc. $\mathrm{cu} /$ ?page_id=23

- Universidad de Salamanca. (2015). http://www.usal.es/. Obtenido de http:/ / www.usal.es/estrategiasinvestigacion

- Universidad de Talca -,Chile. (2015). www.utalca.cl. Obtenido de http:/ / admision.utalca.cl/ carreras_ todas.html

- Universidad del Desarrollo del Estado de Puebla - UNIDES,- México. (2014). http://universidades.estudia.com. $m x /$. Obtenido de http:// www.unides.edu.mx/

- Universidad del Valle. (2015). http://www.univalle.edu.co/. Obtenido de http:/ / viceinvestigaciones.univalle. edu.co/

- Universidad Internacional SEK-Quito. (2015). www.uisek.edu.ec. Obtenido de https:/ / www.uisek.edu.ec/ es/investigaci\%C3\%B3n/reglamentos-y-premios

- Universidad Mayor de San Andrés. (2014). http://www.umsa.bo/web/guest. Obtenido de http:// dipgis.umsa. bo/?cat $=12$ 
- Universidad Nacional Autónoma de México. (2015). https://www.unam.mx/. Obtenido de https://www. unam.mx/investigacion/institutos-centros-y-programas

- Universidad Nacional de Colombia. (2014). http://unal.edu.co/. Obtenido de http:/ / unal.edu.co/menuprincipal/investigacion/la-investigacion-en-la-universidad/

- Universidad Nacional del Centro de la Provincia de Buenos Aires. (2015). http://www.unicen.edu.ar/. Obtenido de http:/ / www.unicen.edu.ar/node/ 8465

- Universidad Pedagógica Experimental Libertador. (2015). www.upel.edu.ec.

- Universidad Politécnica del Estado de Morelos. (2015). http://www.upemor.edu.mx/. Obtenido de http:// www.upemor.edu.mx/\#

- Universidad Politécnica Salesiana. (2015). www.ups.edu.ec. Obtenido de http:// www.ups.edu.ec/web/guest /grupos-de-investigacion

- Universidad San Francisco de Quito. (2015). www.usfq.edu.ec. Obtenido de https:/ / www.usfq.edu.ec/ investigacion_y_creatividad/Paginas/default.aspx

- Universidad San Martín de Porres. (2014). http://www.usmp.edu.pe/. Obtenido de http:/ / www.usmp.edu.pe/ index.php?pag=nuesuniv\&sec=reglamentos\#

- Universidad Tecnológica Equinoccial. (28 de enero de 2015). Resolución Consejo Universitario. Reglamento de Titulación de Grado. Quito.

- University of Miami. (2015). www.miami.edu. Obtenido de http:// www.miami.edu/research/index.html

- University of Bremen. (2015). http://www.uni-bremen.de/en.html. Obtenido de http://www.uni-bremen.de/en / research.html

- Vera, M. (2015). Gestión de la Responsabilidad Social Universitaria. En La Facultad De Ciencias Económica y Negocios, Universidad Tecnológica Equinoccial, Sede Matriz. 2014-2015. Tesis de Maestría Gestión Empresarial. Loja: UTPL. 